\title{
Methanol extract from radix of Glycyrrhizae uralensis attenuate methamphetamine-induced hyperlocomotor activity
}

\author{
ZhengLin Zhao ${ }^{1} \cdot$ Yan Wang ${ }^{1} \cdot$ Feng Lin ${ }^{1} \cdot$ Hui Fu ${ }^{1} \cdot$ FuBo Zhou ${ }^{1} \cdot$ Suchan Chang ${ }^{2} \cdot$ Nu Ri Han ${ }^{2}$ \\ - Dae Hwa Jung ${ }^{2} \cdot$ Chae Ha Yang ${ }^{2} \cdot$ Sang Chan Kim ${ }^{2 \#^{*}} \cdot$ RongJie Zhao ${ }^{1,2 \#^{*}}$ \\ 1Department of Pharmacology, Mudanjiang Medical University, Mudanjiang, \\ China $\cdot{ }^{2}$ MRC-GHF, Daegu Haany University, Gyeongsan, Korea

\section{감초 메탄올 추출물의 메스암페타민 유도 과다보행활동에 대한 억제작용} \\ 자오정린 ${ }^{1} \cdot$ 왕옌 $^{1} \cdot$ 린휭 $^{1} \cdot$ 후우후이 $^{1} \cdot$ 저우후우쀠 $^{1} \cdot$ 장수찬 $^{2} \cdot$ 한누리 $^{2} \cdot$ 정 대화 $^{2} \cdot$ \\ 양재하 ${ }^{2} \cdot$ 김상찬 $^{2 \# *} \cdot$ 자오르옹지에 ${ }^{1,2 \# *}$ \\ ${ }^{1}$ 중국 목단강의과대학 약리학교실 · ${ }^{2}$ 대한민국 대구한의대학교 $\mathrm{MRC}-\mathrm{GHF}$
}

\begin{abstract}
Background and objective: Methamphetamine (Meth) is a widely abused psychostimulant that produces hyperlocomotion in rodents. Radix of Glycyrrhizae uralensis comprises a variety of bioactive components that have neuroprotective effects. In a previous study, we have demonstrated methanol extracts from radix of Glycyrrhizae uralensis (MEGR) suppress acute cocaine-induced extracellular dopamine release in the nucleus accumbens. In the present study, we investigated the effect of MEGR on acute Meth-induced hyperlocomotion.

Methods: Male Sprague - Dawley rats were orally administered with MEGR $(60 \mathrm{mg} / \mathrm{kg}$ and $180 \mathrm{mg} / \mathrm{kg}) 60$ min prior to an intraperitoneal injection of Meth $(1.0 \mathrm{mg} / \mathrm{kg})$.

Results: Behavioral analysis showed acute Meth greatly increased locomotor activities, while pretreatment

(c) 2014 The Korean Medicine Society For The Herbal Formula Study

This paper is available at http/l:www.ompak.okdanche.com which permits unrestricted non-commercial use, distribution, and reproduction in any medium, provided the original work is properly cited.
\end{abstract}


with MEGR dose dependently inhibited the hyperlocomotion. In parallel, there were markedly increased levels of dopamine and its metabolite 3, 4-dihydroxyphenylacetic acid in the nucleus accumbens tissues in Meth-treated rats, which were also almost completely reversed by $180 \mathrm{mg} / \mathrm{kg} \mathrm{MEGR}$.

Conclusions: These results showed that radix of Glycyrrhizae uralensis attenuates Meth-induced hyperlocomotion by inhibiting dopamine synthesis and utilization, suggesting that radix of Glycyrrhizae uralensis might be effective in blocking the rewarding effect of Meth.

Keyword : Methamphetamine, Glycyrrhizae uralensis, Locomotion, Dopamine, Nucleus accumbens

\section{I . Introduction}

Although methamphetamine (Meth) dependence is a serious worldwide public health problem with major medical, psychiatric, socioeconomic and legal consequences [1], there is currently no FDA approved pharmacotherapy for this addictive disorder. The rewarding effects of Meth including euphoria and many other psychostimulant effects are the mainstay to sustain the continued use of Meth, ultimately lead to Meth addition [2]. Therefore, nullifying the rewarding effects of Meth is a promising approach to treat Meth addition.

It is well documented that the commonly abused psychostimulants including Meth increase locomotor activity in rodents, and the biological mechanisms of this stimulant action are homologous with their rewarding effects [3]. Convincing evidence shows that the rewarding effects of Meth mainly come from enhanced dopaminergic neurostransmissions in the mesolimbic and mesocortical brain regions such as the ventral tegmental area, the nucleus accumbens (Nacc) and the medial prefrontal cortex, which also mediate Meth-induced hyperlocomotion [4]. Therefore, inhibition on the increased dopaminergic activities in the mesolimbic brain regions biochemically prevents the formation of the biological bases for Meth addiction and behaviorally blocks hyperlocomotion induced by Meth. For example, a selective dopamine (DA) 1 receptor antagonist SCH23390 injection into the Nacc attenuates Meth-induced hyperlocomotion in rats [5], Limonene inhibits Meth-induced increased locomotor activity via regulation of enhanced accumbal DA release [6].

Radix of Glycyrrhizae uralensis (G. radix), one of the oldest and most popularly used botanicals in traditional Oriental medicine, contains flavonoids and pentacyclic triterpene saponin as major constituents, which include liquiritigenin, isoliquiritigenin, liquiritin, liquiritin apioside, and glycyrrihizin [7]. Being an important tonic, G. radix is generally used to replenish and invigorate deficient Qi and blood, and widely recommended for life-enhancing properties and cure of injury or swelling and for detoxification [8]. Historically, most experimental studies on pharmacological values of $G$. radix were devoted to its anti-inflammatory and anti-oxidative properties [9, 10], however, over the last decade, numerous reports from various laboratories have emphasized the neuropharmacological properties of G. radix, and these reports point to the

\footnotetext{
\# Authors contributed equally.

* Corresponding author : Sang Chan Kim, Daegu Haany University, \#1, Hanuidae-ro, Gyeongsan-si, Gyeongsangbuk-do, 712-715, South Korea.

- Tel : 82-53-819-1863

·E-mail: sckim@dhu.ac.kr

RongJie Zhao, Mudanjiang Medical University, \#3 Tongxiang Street, Mudanjiang, China,

- Tel : 86-453-2984682

·E-mail: zhao_rongjie@yahoo.com

- Received : May 8, 2014 / Revised : May 30, 2014 / Accepted : June 7, 2014
} 
neuroprotective effects of G. radix and its bioactive ingredients against various noxious stimuli [11]. Most notably, studies done in our lab have shown that methanol extracts of G. radix (MEGR) and isoliquiritigenin suppress cocaine-induced extracellular dopamine release in rat Nacc through GABAb receptors [12], and isoliquiritigenin also prevents Meth-induced neurotoxicity in mouse brain by inhibiting $\mathrm{NF}-\mathrm{kB}$ activation [13]. These observations conjunction with its neuroprotective effects reported by others lead to a hypothesis that G. radix have preventive effects against Meth-induced psychostimulant effects.

To test this hypothesis, in the present study we evaluate the effects of MEGR on locomotor activities and accumbal DA and 3, 4dihydroxyphenylacetic acid (DOPAC) levels in rats following an acute Meth administration.

\section{ПI. Materials and methods}

\section{Preparation of MEGR}

The air-dried G. radix was purchased from Daewon Pharmacy (Daegu, Republic of Korea), and its identity and composition was confirmed by Professor Sang Chan Kim of the College of Oriental Medicine at Daegu Haany University in Korea. The G. radix was crushed into powder and extracted with methanol three times for 12 $\mathrm{h}$ each at room temperature. The extract was filtered through a $0.2 \mu \mathrm{m}$ filter (Nalgene, NY, USA), evaporated under reduced pressure at temperature of $37{ }^{\circ} \mathrm{C}$ and lyophilized. The amount of extracts was estimated from the dry weight of lyophilized powder, and the yield was 15.39\%, and the high performance liquid chromatography (HPLC) fingerprint of MEGR is shown in Fig. 1. The MEGR was dissolved in 5\% Tween-80 and orally administered.

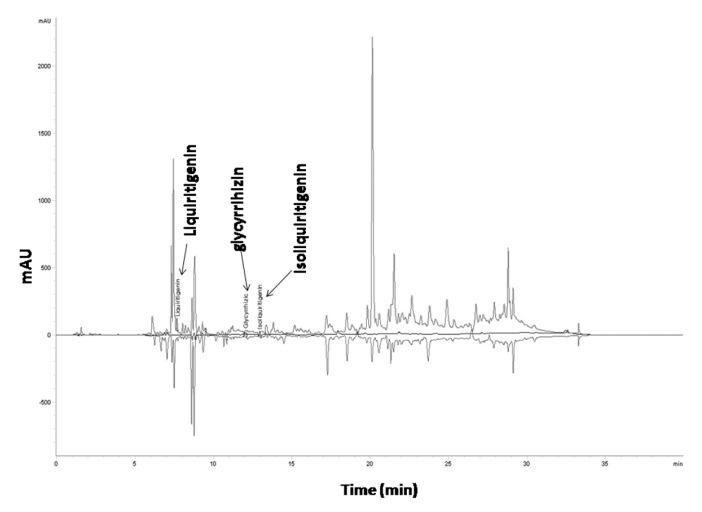

Figure 1. The HPLC profiles of MEGR at $254 \mathrm{~nm}$ (liquiritigenin), $276 \mathrm{~nm}$ (glycyrrhizic acid), and $380 \mathrm{~nm}$ (isoliquiritigenin).

Flow rate: $1.0 \mathrm{~mL} / \mathrm{min}$, column: Waters $\mathrm{XTerrat}^{\mathrm{TM}} \mathrm{RP} 18(150$ $\times 4.6 \mathrm{~mm}, 5 \mu \mathrm{m})$

\section{Reagents}

Sodium octanesulfonic acid acetonitrile, tetrahydrofurane, DA and DOPAC were purchased from Sigma Co. (St Louis, MO, USA). All other drugs were of analytical or HPLC grade.

\section{Animals and experimental design}

Adult male Sprague-Dawley rats (250-270 g) were obtained from Hyochang Science (Hyuchang, Pusan, South Korea), and housed under a controlled environment with food and water ad libitum throughout the course of the study. All animal procedures were approved by the Institutional Animal Care and Use Committee for the National Institutes of Health guidelines concerning the Care and Use of Laboratory Animals.

Meth (purity > 98\%, obtained from Korean Food and Drug Administration) was dissolved in saline and administered intraperitoneally (i.p.) to rats in a single dose of $1.0 \mathrm{mg} / \mathrm{kg}$. MEGR (60 and $180 \mathrm{mg} / \mathrm{kg}$ ) or the vehicle was acutely applied to rats 60 min prior to Meth administration. After MEGR (or vehicle) administration, each rat was allowed to adapt the locomotor testing box for $60 \mathrm{~min}$ followed by Meth administration, 


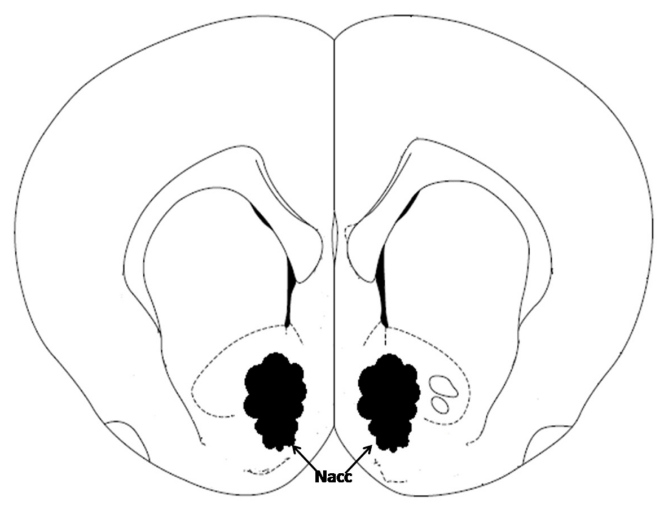

Figure 2. Diagrammatic representation of a coronal section showing the Nacc.

and immediately after the Meth the locomotor activity of each rat was measured in the box for $60 \mathrm{~min}$. The box used to measure locomotor activity is a rectangular container $(40 \mathrm{x} 40 \mathrm{x}$ $45 \mathrm{~cm}$ ) equipped with a video camera above the center of the floor. The walls and floor were made of a clear plexiglas and painted black. The ambulatory distance was recorded by a videotracking system using the Ethovision program (NoldusInformation Technology BV, Wageningen, Netherlands).

\section{DA and DOPAC analysis}

Immediately after the behavioral test, each rat was decapitated and the entire brain was moved and stored at $-80^{\circ} \mathrm{C}$. To determine the effects of MEGR on the Meth-induced changes of mesolimbic dopaminergic activities, the accumbal DA and DOPAC concentrations were measured using HPLC analysis. The stored brain was dissected using the coordinates of Paxinos and Watson [14] to obtain Nacc tissues(Fig. 2). The tissues were sonicated in $1 \mathrm{ml}$ of $0.1 \mathrm{M} \mathrm{HClO}_{4}$ for $30 \mathrm{~s}$, and centrifuged for $15 \mathrm{~min}$ at 26,000 $\mathrm{g}$, $4^{\circ} \mathrm{C}$. Then, a $20-\mu \mathrm{l}$ supernatant aliquot was injected directly into the HPLC with a coulmoetric detector (Coulochem II; ESA, Bedford, MA, USA). The
HPLC system consists of a C18 reverse-phase column(5 u ODS; Altex, Ann Arbor, MI, USA) and an electrochemical transducer with a glassy carbon electrode set at $350 \mathrm{mV}$. The mobile phase was $0.16 \mathrm{M}$ citric acid, $\mathrm{pH}$ 3.0, containing 0.02 mM EDTA with $0.69 \mathrm{mM}$ sodium octanesulfonic acid as an ion-pairing reagent, $4 \%(\mathrm{v} / \mathrm{v})$ acetonitrile and $1.7 \%(\mathrm{v} / \mathrm{v})$ tetrahydrofurane. Peaks and values of DA and DOPAC in samples were identified and calculated by comparing their retention times and peak heights with those of standards. Results were reported as $\mathrm{ng} / \mathrm{g}$ protein. The protein concentration in brain homogenate was determined by the BCA protein assay.

\section{Statistical analysis}

All data were expressed as means \pm standard error of the mean (SEM) and statistically analyzed by one-way analysis of variance (ANOVA) followed by the Newman-Keuls multiple comparison test using the commercially available GraphPad Prism 5.0 software (GraphPad Software, San Diego, CA, USA). A $P$ value $<0.05$ was considered to indicate statistical significance.

\section{Results}

\section{Effects of MEGR on Meth-induced locomotor activity}

Figure. 3 shows the effect of MEGR on locomotor activities. ANOVA on data from MEGR treatment revealed a significant effect $[\mathrm{F}(3,20)=17.8, \mathrm{p}<0.001]$. Post hoc comparisons revealed acute Meth significantly increased locomotor activities in rats (vehicle+saline vs. vehicle+Meth, $\mathrm{p}<0.001$ ), while pretreatment with both doses of MEGR significantly inhibited the Meth-induced hyperlocomotion (vehicle+Meth 


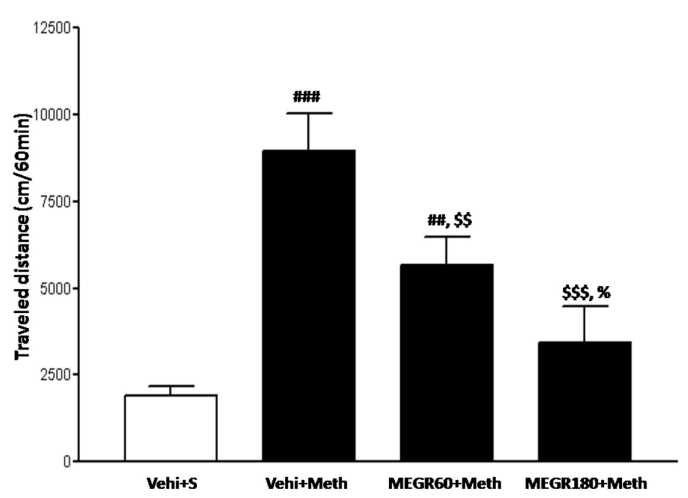

Figure 3. Effects of MEGR on acute Meth-induced hyperlocomotion. The traveled distances were measured for 60 min immediately after Meth.

Data represent mean \pm SEM ( $n=6 /$ group). Vehi: vehicle, S: saline, ${ }^{\# \#} p<0.001,{ }^{\#} p<0.01$, compared with vehicle-treated control group; ${ }^{\$ \$ \$} p<0.001,{ }^{\$ \$} p<0.01$, compared with Meth-treated control group; $\% p<0.05$, compared with MEGR60+Meth group by post hoc test.

vs. MEGR60, $\mathrm{p}<0.01$; vehicle+Meth vs. MEGR180, $\mathrm{p}<0.01$ ), and the effects showed a dosedependent manner (MEGR60+Meth vs. MEGR180+ Meth, $\mathrm{p}<0.05)$. In addition, the only MEGR treated group did not exhibit any change in locomotor activity compared with vehicle control group (Fig. 4). Thus, MEGR might exert the suppressive effect specifically on Meth-induced hyperlocomotion.

\section{Effects of MEGR on Meth-induced changes} of accumbal DA and DOPAC concentrations.

Fig. 5. shows the effects of MEGR on accumbal levels of DA and DOPAC. ANOVA and post hoc analysis showed a single dose of Meth $(1 \mathrm{mg} / \mathrm{kg})$ significantly increased both DA $[\mathrm{F}(3,20)=6.2, \mathrm{p}<0.01$; vehicle + saline vs. vehicle+Meth, $\mathrm{p}<0.01]$ and $\mathrm{DOPAC}[\mathrm{F}(3,20)$ $=7.5, \mathrm{p}<0.01$; vehicle+saline vs. vehicle+Meth, $\mathrm{p}<0.01]$ concentrations in the Nacc, indicating the enhanced synthesis and utilization of DA. However, similar to the behavioral results, pretreatment with $180 \mathrm{mg} / \mathrm{kg}$ MEGR significantly

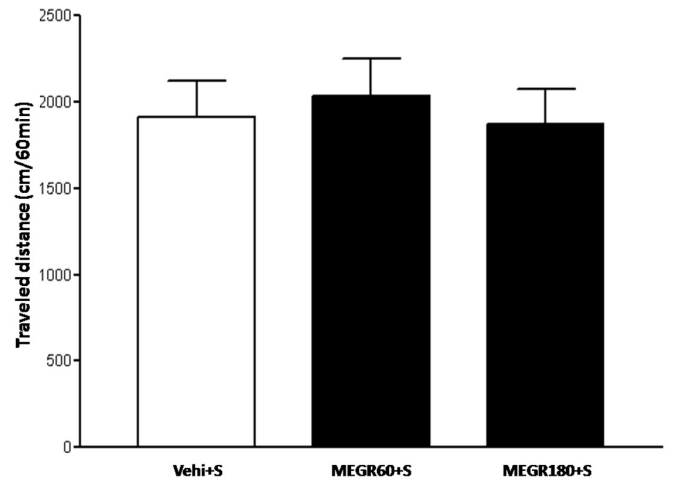

Figure 4. Effects of MEGR on the locomotor activities of saline-treated rats.

Rats were treated with a single dose of saline $60 \mathrm{~min}$ after an acute $60 \mathrm{mg} / \mathrm{kg}$ or $180 \mathrm{mg} / \mathrm{kg}$ MEGR, and then the traveled distance of each rat was measured for 60 min immediately after the saline. Data represent mean \pm SEM ( $n=6 /$ group). Vehi: vehicle, $S$ : saline. Statistical analysis showed no difference among the groups.

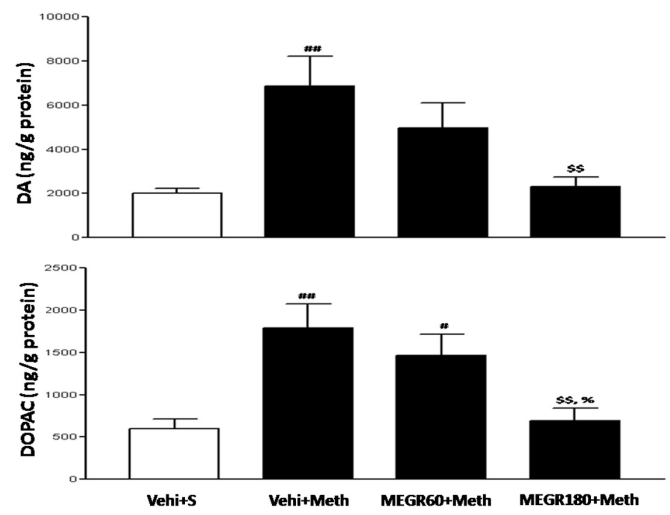

Figure 5. Effects of MEGR on acute Meth-induced changes of accumbal DA and DOPAC concentrations.

The concentrations were measured by HPLC analysis. Data represent mean \pm SEM ( $n=6 /$ group). Vehi: vehicle, $S$ : saline, ${ }^{\#} p<0.01,{ }^{\#} p<0.05$, compared with vehicletreated control group; ${ }^{\$ \$} p<0.01$, compared with Methtreated control group; $\% p<0.05$, compared with MEGR60+ Meth group by post hoc test.

inhibited the increase of both DA (vehicle+Meth vs. MEGR180+Meth, $\mathrm{p}<0.01$ ) and DOPAC (vehicle+Meth vs. MEGR180+Meth, $\mathrm{p}<0.01$ ) levels, while $60 \mathrm{mg} / \mathrm{kg}$ MEGR presented no significant effects (DA: vehicle+Meth vs. MEGR60+Meth, p $>0.05$; DOPAC: vehicle+Meth vs. MEGR60+Meth, 
$\mathrm{p}>0.05)$. In addition, HPLC analysis also showed MEGR itself did not produce any significant impact upon accumbal DA and DOPAC levels (data not shown).

\section{Discussion}

In a previous study we demonstrated MEGR suppresses cocaine-induced extracellular dopamine release in rat Nacc [12], in the present study we extended this result showing that MEGR inhibits acute Meth-induced hyperlocomotion in rats. In the current study, the behavioral experiment revealed pretreatment with $60 \mathrm{mg} / \mathrm{kg}$ and 180 mg/kg MEGR dose--dependently attenuated Methinduced enhanced locomotor activities in rats, in addition, the neurochemical analysis exhibited 180 $\mathrm{mg} / \mathrm{kg}$ MEGR almost completely blocked the acute Meth-induced increase of accumbal DA and DOPAC levels. These findings suggest that MEGR blocks Meth-induced hyperlocomtion through inhibiting elevated DA synthesis and utilization in the Nacc.

Convincing evidence has highlighted the pharmaceutical values of extracts from traditional Oriental herbals in treating abused drug disorders, in particular, several laboratories have reported extracts of tonic herbals have preventive effects against the rewarding effects of abused drugs [15]. For example, Kim et al. [16] have reported ginsenosides $\mathrm{Rb} 1$ and $\mathrm{Rg} 1$ blocks Meth-induced behavioral hyperactivity and conditioned place preference (CPP) in rats, Mattioli et al. [17] have demonstrated a Rhodiola rosea L. hydroalcoholic extract prevents establishment of morphine-induced CPP and facilitates extinction of established CPP. In the present study pretreatment with MEGR prevents Meth-induced hyperlocomotion related to the rewarding effects of Meth. This result suggests that MEGR may have therapeutic effects against Meth addition.

Administration of Meth increases extracellular DA levels [18] in the Nacc by (1) up-regulating tyrosine hydroxylase, the rate-limiting step in DA synthesis, (2) inducing reverse transport of monoamines, (3) blocking the activity of the intracellular vesicular monoamine transporter 2 , (4) decreasing the expression of the DA transporter (DAT) at the cell surface [19, 20]. The released DA activates DA receptors on accumbenal GABAergic medium spiny neurons and promotes sensitivities of DA receptors, which appear to be the main biological basis for Meth-induced hyperlocomotion and rewarding effects [21]. Therefore, either inhibiting DA release or dampening DA sensitivity in the Nacc can counteract psychostimulant effects of Meth on locomotion, further abrogate rewarding effects of Meth. In the above mentioned study, Kim et al. have also demonstrated the therapeutic effect of ginsenosides on Meth-induced behavioral abnormalities is mediated by inhibiting accumbenal postsynaptic DA receptors supersensitivity [16]; in another study, Egecioglu et al. have reported the glucagonlike peptide 1 agonist exendin-4 attenuates cocaine-induced hyperlocomotion by reducing accumbal DA release [22]. In a previous study we demonstrated that MEGR can suppress acute cocaine-induced increase of extracellular DA release in the Nacc [12]. In the current study, we found pretreatment with MEGR almost completely reversed acute Meth-induced enhanced DA synthesis and utilization in rat accumbal tissues. Taken together, these results suggest that the blockade effect of MEGR on Meth-induced hyperlocomotion may be mediated through inhibiting accumbenal DA synthesis and utilization.

In summary, in the present study, we have found pretreatment with MEGR significantly 
attenuates acute Meth-induced hyperlocomotion and inhibits Meth-induced enhanced DA synthesis and utilization in the Nacc. These results suggest that MEGR may be effective in treating Meth addiction, possibly by modulating DAergic neurotransmissions in the Nacc.

\section{Acknowledgements}

This study was supported by the National Research Foundation of Korea (NRF) funded by Korea government (MSIP) (No. 2011-0030124) and the Natural Science Foundation of Heilongjiang Province for Returned Scholars, China (LC201028).

\section{References}

1. Gururajan A, Manning EE, Klug M, van den Buuse M. Drugs of abuse and increased risk of psychosis development. Aust N Z J Psychiatry. 2012; 46 (12): 1120-1135.

2. Sofuoglu M. Cognitive enhancement as a pharmacotherapy target for stimulant addiction. Addiction. 2010; 105 (1): 38-48.

3. Wise RA, Bozarth MA. A psychomotor stimulant theory of addiction. Psychol Rev. 1987; 94 (4): 469-492.

4. Di Chiara G, Tanda G, Bassareo V, Pontieri F, Acquas E, Fenu S, Cadoni C, Carboni E. Drug addiction as a disorder of associative learning. Role of nucleus accumbens shell/extended amygdala dopamine. Ann N Y Acad Sci. 1999; 877: 461-485.

5. Koshikawa N, Mori E, Oka K, Nomura H, Yatsushige N, Maruyama Y. Effects of SCH23390 injection into the dorsal striatum and nucleus accumbens on methamphetamine-induced gnawing and hyperlocomotion in rats. J Nihon Univ
Sch Dent. 1989; 31 (2): 451-457.

6. Yun J. Limonene inhibits methamphetamineinduced locomotor activity via regulation of 5-HT neuronal function and dopamine release. Phytomedicine. Phytomedicine. 2014; 21 (6): 883-887.

7. Kamei J, Nakamura R, Ichiki H, Kubo M. Antitussive principles of Glycyrrhizae radix, a main component of the Kampo preparations Bakumondo-to (Mai-mendong-tang). Eur. J. harPmacol. 2003; 469: 159 - 163.

8. Wang ZY, Nixon DW. Licorice and cancer, Nutr. Cancer 2001; 39: 1 - 11.

9. Kim YW, Zhao RJ, Park SJ, Lee JR, Cho IJ, Yang CH, Kim SG, Kim SC. Anti-inflammatory effects of liquiritigenin as a consequence of the inhibition of NF-kappaB-dependent iNOS and proinflammatory cytokines production. $\mathrm{Br}$ J Pharmacol. 2008; 154 (1): 165-173.

10. Kim SC, Park SJ, Lee JR, Seo JC, Yang $\mathrm{CH}$, Byun SH. Cytoprotective activity of glycyrrhizae radix extract against arsenite-induced cytotoxicity. Evid Based Complement Alternat Med. 2008; 5(2): 165-171.

11. Shishkina GT, Dygalo NN, Yudina AM, Kalinina TS, Tolstikova TG, Sorokina, IV, Kovalenko IL, Anikina LV. The effects of fluoxetine and its complexes with glycerrhizic acid on behavior in rats and brain monoamine levels. Neurosci. Behav. Physiol. 2006; 36: $329-333$.

12. Jang EY, Choe ES, Hwang M, Kim SC, Lee JR, Kim SG, Jeon JP, Buono RJ, Yang $\mathrm{CH}$. Isoliquiritigenin suppresses cocaine-induced extracellular dopamine release in rat brain through GABA(B) receptor. Eur J Pharmacol. 2008; 587 (1-3): 124-128.

13. Lee MJ, Yang $\mathrm{CH}$, Jeon JP, Hwang $\mathrm{M}$. Protective effects of isoliquiritigenin against methamphetamine-induced neurotoxicity in 
mice. J Pharmacol Sci. 2009; 111 (2): 216220.

14. Paxinos G, Watson C. The Rat Brain in Stereotaxic Coordinates, The third ed. Academic Press 1997, New York.

15. Lu L, Liu Y, Zhu W, Shi J, Liu Y, Ling W, Kosten TR. Traditional medicine in the treatment of drug addiction. Am J Drug Alcohol Abuse. 2009; 35 (1): 1-11.

16. Kim HS, Hong YT, Oh KW, Seong YH, Rheu HM, Cho DH, Oh S, Park WK, Jang CG. Inhibition by ginsenosides Rb1 and Rg1 of methamphetamine-induced hyperactivity, conditioned place preference and postsynaptic dopamine receptor supersensitivity in mice. Gen Pharmacol. 1998; 30 (5): 783-789.

17. Mattioli L, Titomanlio F, Perfumi M. Effects of a Rhodiola rosea L. extract on the acquisition, expression, extinction, and reinstatement of morphine-induced conditioned place preference in mice. Psychopharmacology (Berl). 2012; 221 (2): 183-193.

18. Zhang Y, Loonam TM, Noailles PA, Angulo JA. Comparison of cocaine- and methamphetamineevoked dopamine and glutamate overflow in somatodendritic and terminal field regions of the rat brain during acute, chronic, and early withdrawal conditions. Ann N Y Acad Sci. 2001; 937: 93-120.

19. Fleckenstein AE, Volz TJ, Riddle EL, Gibb JW, Hanson GR. New insights into the mechanism of action of amphetamines. Annu Rev Pharmacol Toxicol. 2007; 47: 681-698.

20. Karila L, Weinstein A, Aubin HJ, Benyamina A, Reynaud M, Batki SL. Pharmacological approaches to methamphetamine dependence: a focused review. Br J Clin Pharmacol. 2010; 69 (6):578-592.

21. Rose ME, Grant JE. Pharmacotherapy for methamphetamine dependence: a review of the pathophysiology ofmethamphetamine addiction and the theoretical basis and efficacy of pharmacotherapeutic interventions. Ann Clin Psychiatry. 2008; 20 (3): 145-155.

22. Egecioglu E, Engel JA, Jerlhag E. The glucagon-like peptide 1 analogue, exendin-4, attenuates the rewarding properties of psychostimulant drugs in mice. PLoS One. 2013; 8 (7): e69010. doi: 10.1371/joumal.pone.0069010. 